\title{
AN INDEX FORMULA FOR LOEWNER VECTOR FIELDS
}

\author{
FreDERICO XAVIER
}

\begin{abstract}
Let $f$ be $C^{2}$ real-valued function defined near 0 in $\mathbb{R}^{2}, \frac{\partial^{2} f}{\partial \bar{z}^{2}} \neq 0$ for $z \neq 0$. Motivated by the Carathéodory conjecture in differential geometry, Loewner conjectured that the index at 0 of the vector field given in complex notation by $\frac{\partial^{2} f}{\partial \bar{z}^{2}}$ is at most two. In this paper we establish a formula that computes the index of these Loewner vector fields from data about the hessian of $f$.
\end{abstract}

\section{$\S 1$ Introduction.}

The well-known Carathéodory conjecture in classical differential geometry states that any smoothly immersed sphere in $\mathbb{R}^{3}$ must have at least two umbilical points. Several solutions to this problem have been proposed over the years, all in the case of analytic immersions [1], [5], [6], [8], [9], [14] (for related work on umbilics, see for instance [4], [10], $[11],[12])$. In these papers the aim was to prove a stronger form of the Carathéodory conjecture namely, that the local index of the fields of lines of curvature near an isolated umbilic cannot exceed one (since $\chi\left(S^{2}\right)=2$, in an immersed sphere with a single umbilical point the fields of principal directions would have index two, a contradiction to the conclusion in the local conjecture).

The local approach followed in [1], [5], [6], [8], [9], [14] involves an equivalent conjecture attributed to Loewner (sometimes also to $\mathrm{Bol}$ ), about the way the square of the Cauchy-Riemann operator acts on real-valued functions. Let $B$ be the open unit ball in $\mathbb{R}^{2}$ centered at $0, T=\partial B$,

$$
\frac{\partial}{\partial \bar{z}}=\frac{1}{2}\left(\frac{\partial}{\partial x}+i \frac{\partial}{\partial y}\right), \quad \frac{\partial^{2}}{\partial \bar{z}^{2}}=\frac{1}{4}\left(\frac{\partial^{2}}{\partial x^{2}}-\frac{\partial^{2}}{\partial y^{2}}+2 i \frac{\partial^{2}}{\partial x \partial y}\right)
$$

The Loewner Conjecture. If $f \in C^{2}(\bar{B}, \mathbb{R})$ and $\frac{\partial^{2} f}{\partial \bar{z}^{2}} \neq 0$ for $z \neq 0$, then the index at zero of the vector field given in complex notation by $\frac{\partial^{2} f}{\partial \bar{z}^{2}}$ satisfies Ind $\left(\frac{\partial^{2} f}{\partial \bar{z}^{2}}, 0\right) \leq 2$.

It is commonly assumed that the function in the Loewner conjecture is defined on a neighborhood of 0 but, by a dilation, there is no loss of generality in taking the domain to be the unit disc. Since the index of $\frac{\partial^{2} f}{\partial \bar{z}^{2}}$ remains constant under small $C^{2}$ perturbations of $f$ near $T$, it may also be assumed that $f$ is of class $C^{3}$ near $T$.

Received by the editors October 30, 2006. 
The Loewner vector field $\frac{\partial^{2} f}{\partial \bar{z}^{2}}$ can be identified (up to a constant) with the traceless part of the hessian matrix $H_{f}$ of $f$, so that the non-vanishing condition in the Loewner conjecture implies that $H_{f}$ is not a multiple of the identity for $z \neq 0$. Thus, at a point other than the origin $H_{f}$ has precisely two diagonalizing directions. This gives rise to two orthogonal one-dimensional foliations, possibly unorientable and with a singularity at 0 . The geometric content of the conjecture is that the index at 0 of either of these fields of eigendirections of $H_{f}$ is a half-integer that cannot exceed one (the index of $\frac{\partial^{2} f}{\partial \bar{z}^{2}}$ is always twice the index of the fields of eigendirections of $H_{f}$, see [12]).

Motivated by the $C^{2}$ Loewner Conjecture, in this paper we introduce a technique that allows us to compute the index of $\frac{\partial^{2} f}{\partial \bar{z}^{2}}$ in terms of the eigenvalues and eigenvectors of $H_{f}$ along $T$ :

Theorem 1 (An index formula for Loewner vector fields). Let $f \in C^{2}(\bar{B}, \mathbb{R})$ be $C^{3}$ near $T, \frac{\partial^{2} f}{\partial \bar{z}^{2}} \neq 0$ for $z \neq 0$. Denote by $\lambda, \mu, \lambda \geq \mu$, the eigenvalues of $H_{f}$, so that $\lambda>\mu$ for $z \neq 0$. Let $\Sigma=\Sigma_{\lambda} \cup \Sigma_{\mu}$, where $\Sigma_{\lambda}$ (resp., $\Sigma_{\mu}$ ) is the set of points $p \in T$ for which $H_{f}(p) p=\lambda(p) p$ (resp., $\left.H_{f}(p) p=\mu(p) p\right)$. We write $\frac{\partial}{\partial r}$ for the radial derivative and assume that the function $\lambda-\mu-\frac{\partial \mu}{\partial r}$ (resp., $\mu-\lambda-\frac{\partial \lambda}{\partial r}$ ) has no zeros on $\Sigma_{\lambda}$ (resp., $\left.\Sigma_{\mu}\right)$. Then $\Sigma$ is finite and, furthermore,

$$
\begin{aligned}
\text { Ind } & \left(\frac{\partial^{2} f}{\partial \bar{z}^{2}}, 0\right) \\
= & 2+\#\left\{p \in \Sigma_{\lambda},\left(\lambda-\mu-\frac{\partial \mu}{\partial r}\right)(p)<0\right\}-\#\left\{p \in \Sigma_{\lambda},\left(\lambda-\mu-\frac{\partial \mu}{\partial r}\right)(p)>0\right\} \\
& =2+\#\left\{p \in \Sigma_{\mu},\left(\mu-\lambda-\frac{\partial \lambda}{\partial r}\right)(p)>0\right\}-\#\left\{p \in \Sigma_{\mu},\left(\mu-\lambda-\frac{\partial \lambda}{\partial r}\right)(p)<0\right\} .
\end{aligned}
$$

Remarks. Despite the conspicuous presence of the number two in it, taken by itself the index formula above does not seem to either support or contradict the validity of the Loewner Conjecture. The second formula follows from the first applied to $-f$, but our arguments will yield both formulas simultaneously. The hypothesis on the zeros of $\lambda-\mu-\frac{\partial \mu}{\partial r}$ and $\mu-\lambda-\frac{\partial \lambda}{\partial r}$ is satisfied generically in the $C^{3}$ topology (near $T$, away from the singularity). As already observed, there is no loss of generality in assuming that the function is $C^{3}$ near $T$. If $f \in C^{2}(\bar{B}, \mathbb{R})$ is $C^{3}$-generic near $T$ and $\frac{\partial^{2} f}{\partial \bar{z}^{2}} \neq 0$ for $z \neq 0$, then the index can be estimated from above by $2+\min \left\{\# \Sigma_{\lambda}, \# \Sigma_{\mu}\right\}$. In principle, one (or both) of the sets $\Sigma_{\lambda}, \Sigma_{\mu}$ may be empty.

The main tool used to prove Theorem 1 is the classical Bendixson index formula for two-dimensional singularities, applied to some carefully chosen vector fields. Loosely speaking, the main idea in the present paper is to create a geometric mechanism that allows to transition from the vector field $\frac{\partial^{2} f}{\partial \bar{z}^{2}}$ to the much simpler field $i \frac{\partial f}{\partial \bar{z}}$. We remark that the idea of using Bendixson's formula to count internal and external tangencies of principal foliations was also used in [3]. The essential elements of our proof carry over to the case of a general Riemannian surface when, instead of the hessian of a function, one works with a symmetric tensor satisfying the Codazzi equations. For completeness, 
we recall that a smooth field $A$ of symmetric linear endomorphisms on an abstract Riemannian surface $M$ is said to be a Codazzi field if, relative to the natural covariant derivative $\nabla$ of $M$, one has $\left(\nabla_{X} A\right) Y=\left(\nabla_{Y} A\right) X$ for all vector fields $X$ and $Y$. The main example of a Codazzi field is the second fundamental form of a surface in $\mathbb{R}^{3}$. A comprehensive treatment can be found in [2]. We also refer to [13] for connections between Codazzi tensors and the dynamics of principal foliations.

Acknowledgments. It is a pleasure to thank D. Gromoll and B. Lawson for having invited us to spend a sabbatical leave at Stony Brook, where this project was initiated. Thanks are also due to L.F. Mello for suggestions that led to improvements in the presentation of the paper.

\section{$\S 2$ Proof of Theorem 1.}

Replacing $f$ by $f-\langle\nabla f(0), \cdot\rangle$, we may assume that $\nabla f(0)=0$. For $z \in \mathbb{R}^{2}$ let $g(z)=\frac{|z|^{2}}{2}$, so that $\nabla g(z)=z$ and $H_{g}(z)=I$. Since

$$
\frac{\partial^{2} f}{\partial \bar{z}^{2}}=\frac{\partial^{2}}{\partial \bar{z}^{2}}(f+c g)
$$

we may replace $f$ by $f+c g, c>0$ large, and assume further that $H_{f}>0$ (that is, $H_{f}$ is positive-definite) and 0 is the only critical point of $f$ in $\bar{B}$. It is also easy to see that for large $c>0$ the vector field $\nabla(f+c g)$ is homotopic to $c \nabla g$ through a linear homotopy that vanishes only at $z=0$. In particular, renaming $f+c g$ to be $f$, we may assume

$$
\text { Ind }(\nabla f, 0)=\text { Ind }(\nabla g, 0)=1 \text {. }
$$

One can identify a symmetric traceless $2 \times 2$ matrix $\left(a_{i j}\right)$ with the complex number $a_{11}+i a_{12}$, and so

$$
\nabla f \cong 2 \frac{\partial f}{\partial \bar{z}}, \quad\left(H_{f}-\frac{\Delta f}{2} I\right) \cong 2 \frac{\partial^{2} f}{\partial \bar{z}^{2}}, \quad\left(H_{f}-\frac{\Delta f}{2} I\right) \nabla f \cong 4 \frac{\partial^{2} f}{\partial \bar{z}^{2}} \frac{\partial f}{\partial z}
$$

In view of our normalizations, the identity below is meaningful (we set $k=\frac{\Delta f}{2}$ ):

$$
\begin{aligned}
\operatorname{Ind}\left(\frac{\partial^{2} f}{\partial \bar{z}^{2}}, 0\right)=\operatorname{Ind}\left(\frac{\partial^{2} f}{\partial \bar{z}^{2}}\left|\frac{\partial f}{\partial \bar{z}}\right|^{2}, 0\right)=\operatorname{Ind}\left(\frac{\partial^{2} f}{\partial \bar{z}^{2}} \frac{\partial f}{\partial z}, 0\right) & +\operatorname{Ind}\left(\frac{\partial f}{\partial \bar{z}}, 0\right) \\
& =\operatorname{Ind}\left(\left(H_{f}-k I\right) \nabla f, 0\right)+1
\end{aligned}
$$

Consider the vector fields

$$
X=J H_{f} \nabla g, \quad Y=J\left(H_{f}-k I\right) \nabla g,
$$


where $J$ is counterclockwise rotation by $\frac{\pi}{2}$. Again, renaming $f+c g$ (with $c>0$ large) to be $f$, we may assume

$$
\operatorname{Ind}\left(J\left(H_{f}-k I\right) \nabla f, 0\right)=\operatorname{Ind}\left(J\left(H_{f}-k I\right) \nabla g, 0\right)=\operatorname{Ind}(Y, 0) .
$$

From (2) and (4) one sees that the task of finding a formula for the index of the Loewner vector field $\frac{\partial^{2} f}{\partial \bar{z}^{2}}$ is reduced to the computation of $\operatorname{Ind}(Y, 0)$.

The points in $\Sigma_{\lambda}$ and $\Sigma_{\mu}$ will be referred to as $\lambda$-points and $\mu$-points, respectively. Notice that

$$
H_{f} \nabla g=\lambda \nabla g, H_{f} J \nabla g=\mu J \nabla g
$$

at a $\lambda$-point, and

$$
H_{f} \nabla g=\mu \nabla g, H_{f} J \nabla g=\lambda J \nabla g
$$

at a $\mu$-point.

Using the fact that $J \nabla g$ is tangent to $T$ we arrive at our fundamental observation:

( $\dagger$ ) The subset of $T$ where either $X$ or $Y$ is tangent to $T$ is precisely the set $\Sigma$.

We recall Bendixson's formula for computing the index of an isolated zero $p$ of a smooth planar field $\xi$ ([7], p.173, thm. 9.2). Let $C$ be a smooth positively oriented closed curve bounding an open Jordan domain $D, p \in D$. Assume that $\xi$ is defined on a neighborhood of $\bar{D}$ and that $p$ is its only zero. Suppose that $\xi$ is tangent to $C$ at only finitely many points. A point $q \in C$ where $\xi$ is tangent to $C$ will be called $\xi$-elliptic (resp., $\xi$ - hyperbolic) if there exists $\epsilon>0$ such that the trajectory of $\xi$ passing through $q$ at time zero is contained in $D$ (resp., contained in the complement of $\bar{D}$ ) for all times $t$ with $0<|t|<\epsilon$. Bendixson's index formula asserts that

$$
\operatorname{Ind}(\xi, p)=1+\frac{e-h}{2},
$$

where $e$ and $h$ stand for the number of points in $C$ that are $\xi$-elliptic and $\xi$-hyperbolic, respectively.

We want to apply (5) to both $X$ and $Y$, with the choice $C=T$. Once again, notice the crucial fact that, by $(\dagger)$, the tangencies of $X$ and $Y$ along $T$ occur on the same set. This will enable us to express the index of the vector field $Y$ in terms of quantities associated to $X$.

Let $e_{X}, h_{X}, e_{Y}, h_{Y}$ denote the number of elliptic and hyperbolic points along $\Sigma$, relative to $X$ and $Y$. We refine matters further by considering $\lambda$-points and $\mu$-points. Let then $e_{X}^{\lambda}$ and $h_{X}^{\lambda}$ stand for the number of $X$-elliptic and $X$-hyperbolic points that are also $\lambda$-points. One can introduce in a similar fashion the quantities $e_{X}^{\mu}, h_{X}^{\mu}$, as well 
as $e_{Y}^{\lambda}, h_{Y}^{\lambda}$ and $e_{Y}^{\mu}, h_{Y}^{\mu}$. The following relations hold trivially:

$$
\begin{aligned}
& e_{X}=e_{X}^{\lambda}+e_{X}^{\mu} \\
& h_{X}=h_{X}^{\lambda}+h_{X}^{\mu} \\
& e_{Y}=e_{Y}^{\lambda}+e_{Y}^{\mu} \\
& h_{Y}=h_{Y}^{\lambda}+h_{Y}^{\mu} .
\end{aligned}
$$

The relations below, on the other hand, are not obvious:

$$
\begin{aligned}
& e_{X}^{\lambda}+e_{X}^{\mu}=h_{X}^{\lambda}+h_{X}^{\mu} \\
& e_{X}^{\lambda}=e_{Y}^{\lambda} \\
& h_{X}^{\lambda}=h_{Y}^{\lambda} \\
& e_{X}^{\mu}=h_{Y}^{\mu} \\
& h_{X}^{\mu}=e_{Y}^{\mu} .
\end{aligned}
$$

The first equation in (7) follows from (5) and (6), since $\operatorname{Ind}(X, 0)=1$ (this is so because $H_{f}$ can be deformed into the identity through positive - hence invertible operators).

The proof of the other relations in (7) will involve several computations. The question of whether a particular point is elliptic or hyperbolic, relative to $X$ or $Y$, will be answered by an examination of how the levels of the function $g$ change along the appropriate trajectory. Since the first derivative of $g$ along the trajectory vanishes at time zero because of tangency -, one must compute higher order derivatives. The second order derivatives will be shown to be non-zero at time zero. In particular, the tangency point is hyperbolic or elliptic according to whether the second derivative is positive or negative.

We begin our analysis by considering a local trajectory $\alpha$ of $X=J H_{f} \nabla g$ that is tangent to $T$, so that $\alpha^{\prime}=X(\alpha), \alpha(0)=p \in \Sigma$. In the computations that follow we make use of the fact that the trilinear form $D^{3} f$ is symmetric. This is needed in order to eventually obtain the same $D^{3} f$ terms in (9), (11) and (10), (12) (more generally, if $H_{f}$ is replaced by a symmetric tensor $A$ on a Riemannian surface, one needs the Codazzi equations $\left.\left(\nabla_{X} A\right) Y=\left(\nabla_{Y} A\right) X\right)$. A straightforward calculation yields

$$
\begin{aligned}
& \frac{d^{2}}{d t^{2}} g(\alpha(t))=\left\langle\nabla g, J H_{f} \nabla g\right\rangle^{\prime}=-\left\langle J \nabla g, H_{f} \nabla g\right\rangle^{\prime}= \\
& -\left\langle J H_{g} X, H_{f} \nabla g\right\rangle-D^{3} f(J \nabla g, X, \nabla g)-\left\langle J \nabla g, H_{f} H_{g} X\right\rangle .
\end{aligned}
$$

For future reference, note that $(g \circ \alpha)^{\prime \prime}=X(\langle\nabla g, X\rangle)=\langle X, \nabla\langle\nabla g, X\rangle\rangle$.

Setting

$$
A(p)=\left.\frac{d^{2}}{d t^{2}} g(\alpha(t))\right|_{t=0}
$$

and taking $p=p_{\lambda}$ to be a $\lambda$-point in (8) we have, since $X\left(p_{\lambda}\right)=\lambda J \nabla g\left(p_{\lambda}\right)$,

$$
A\left(p_{\lambda}\right)=-\langle-\lambda \nabla g, \lambda \nabla g\rangle-\lambda D^{3} f(J \nabla g, J \nabla g, \nabla g)-\langle J \nabla g, \lambda \mu J \nabla g\rangle .
$$


Since $|\nabla g|=1$ on $T$ one has, at $p_{\lambda}$,

$$
\frac{A\left(p_{\lambda}\right)}{\lambda}=\lambda-\mu-D^{3} f(J \nabla g, J \nabla g, \nabla g) .
$$

Similarly, at a $\mu$-point $p=p_{\mu}$ we have, using $X\left(p_{\mu}\right)=\mu J \nabla g$,

$$
A\left(p_{\mu}\right)=-\langle-\mu \nabla g, \mu \nabla g\rangle-\mu D^{3} f(J \nabla g, J \nabla g, \nabla g)-\langle J \nabla g, \lambda \mu J \nabla g\rangle,
$$

and so, at $p_{\mu}$,

$$
\frac{A\left(p_{\mu}\right)}{\mu}=\mu-\lambda-D^{3} f(J \nabla g, J \nabla g, \nabla g) .
$$

The function $\left\langle H_{f} J \nabla g, J \nabla g\right\rangle-\mu|\nabla g|^{2}$, defined on a neighborhood of $\bar{B}$, is nonnegative and vanishes precisely at those points where $H_{f} \nabla g=\lambda \nabla g$. Hence any $\lambda$-point $p_{\lambda}$ is an interior critical point for the function in question, and we have (at $p_{\lambda}$ ):

$$
\begin{aligned}
& 0=(\nabla g)\left(\left\langle H_{f} J \nabla g, J \nabla g\right\rangle-\mu|\nabla g|^{2}\right)=D^{3} f(J \nabla g, J \nabla g, \nabla g)+\left\langle H_{f} J \nabla g, J H_{g} \nabla g\right\rangle+ \\
& \left\langle H_{f} J H_{g} \nabla g, J \nabla g\right\rangle-|\nabla g|^{2}\langle\nabla g, \nabla \mu\rangle-2 \mu\left\langle H_{g} \nabla g, \nabla g\right\rangle .
\end{aligned}
$$

In particular,

$$
D^{3} f(J \nabla g, J \nabla g, \nabla g)\left(p_{\lambda}\right)=\langle\nabla g, \nabla \mu\rangle\left(p_{\lambda}\right)=\frac{\partial \mu}{\partial r}\left(p_{\lambda}\right) .
$$

A similar computation with the non-positive function $\left\langle H_{f} J \nabla g, J \nabla g\right\rangle-\lambda|\nabla g|^{2}$ yields, at a $\mu$-point $p_{\mu}$,

$$
D^{3} f(J \nabla g, J \nabla g, \nabla g)\left(p_{\mu}\right)=\langle\nabla g, \nabla \lambda\rangle\left(p_{\mu}\right)=\frac{\partial \lambda}{\partial r}\left(p_{\mu}\right) .
$$

Using (11) and (12), (9) and (10) can be rewritten as

$$
\begin{aligned}
& \frac{A\left(p_{\lambda}\right)}{\lambda\left(p_{\lambda}\right)}=\left(\lambda-\mu-\frac{\partial \mu}{\partial r}\right)\left(p_{\lambda}\right), \\
& \frac{A\left(p_{\mu}\right)}{\mu\left(p_{\mu}\right)}=\left(\mu-\lambda-\frac{\partial \lambda}{\partial r}\right)\left(p_{\mu}\right) .
\end{aligned}
$$

We carry out a similar analysis for the vector field $Y=J\left(H_{f}-k I\right) \nabla g$. Consider a local trajectory $\beta$ of $Y$ that is tangent to $T$, so that $\beta^{\prime}=Y(\beta), \beta(0)=p \in \Sigma$. We compute 


$$
\begin{aligned}
& \frac{d^{2}}{d t^{2}} g(\beta(t))=\left\langle\nabla g, J\left(H_{f}-k I\right) \nabla g\right\rangle^{\prime}=\left\langle\nabla g, J H_{f} \nabla g\right\rangle^{\prime}=-\left\langle J \nabla g, H_{f} \nabla g\right\rangle^{\prime}= \\
& -\left\langle J H_{g} Y, H_{f} \nabla g\right\rangle-D^{3} f(J \nabla g, Y, \nabla g)-\left\langle J \nabla g, H_{f} H_{g} Y\right\rangle
\end{aligned}
$$

If $p=p_{\lambda}$ is a $\lambda$-point, or if $p=p_{\mu}$ is a $\mu$-point,

$$
\begin{array}{ll}
Y\left(p_{\lambda}\right)=(\lambda-k)\left(p_{\lambda}\right) J \nabla g\left(p_{\lambda}\right), & H_{f} Y\left(p_{\lambda}\right)=\mu(\lambda-k)\left(p_{\lambda}\right) J \nabla g\left(p_{\lambda}\right), \\
Y\left(p_{\mu}\right)=(\mu-k)\left(p_{\mu}\right) J \nabla g\left(p_{\mu}\right), & H_{f} Y\left(p_{\mu}\right)=\lambda(\mu-k)\left(p_{\mu}\right) J \nabla g\left(p_{\mu}\right) .
\end{array}
$$

Setting

$$
B(p)=\left.\frac{d^{2}}{d t^{2}} g(\beta(t))\right|_{t=0}
$$

one computes, using (15) and (16),

$$
B\left(p_{\lambda}\right)=-\langle J(\lambda-k) J \nabla g, \lambda \nabla g\rangle-(\lambda-k) D^{3} f(J \nabla g, J \nabla g, \nabla g)-\langle J \nabla g, \mu(\lambda-k) J \nabla g\rangle,
$$

so that

$$
B\left(p_{\lambda}\right)=(\lambda-k)\left(\lambda-\mu-D^{3} f(J \nabla g, J \nabla g, \nabla g)\right) .
$$

Comparing the last expression with (9),

$$
B\left(p_{\lambda}\right)=(\lambda-k) \frac{A\left(p_{\lambda}\right)}{\lambda} .
$$

It follows from (13), (17), $H_{f}>0, k=\frac{\lambda+\mu}{2}$, and the hypotheses of the theorem that $B\left(p_{\lambda}\right)$ and $A\left(p_{\lambda}\right)$ are non-zero and have the same sign. In particular, if $p_{\lambda}$ is $X$ hyperbolic (resp., $X$-elliptic), $p_{\lambda}$ will be $Y$-hyperbolic (resp., $Y$ - elliptic). This gives the relations $h_{X}^{\lambda}=h_{Y}^{\lambda}, e_{X}^{\lambda}=e_{Y}^{\lambda}$ in (7). Finiteness of $\Sigma$ - and hence the finiteness of $e_{X}^{\lambda}, h_{X}^{\lambda}, e_{X}^{\mu}, h_{X}^{\mu}, e_{Y}^{\lambda}, h_{Y}^{\lambda}, e_{Y}^{\mu}, h_{Y}^{\mu}-$, will be established shortly.

From (15) and (16) we evaluate at $p_{\mu}$,

$$
B\left(p_{\mu}\right)=-\langle J(\mu-k) J \nabla g, \mu \nabla g\rangle-(\mu-k) D^{3} f(J \nabla g, J \nabla g, \nabla g)-\langle J \nabla g, \lambda(\mu-k) J \nabla g\rangle,
$$

so that,

$$
B\left(p_{\mu}\right)=(\mu-k)\left((\mu-\lambda)-D^{3} f(J \nabla g, J \nabla g, \nabla g)\right) .
$$

Comparing with (10),

$$
B\left(p_{\mu}\right)=(\mu-k) \frac{A\left(p_{\mu}\right)}{\mu}
$$

In particular, from (14), (18), $H_{f}>0, k=\frac{\lambda+\mu}{2}$, and the hypotheses of the theorem, $B\left(p_{\mu}\right)$ and $A\left(p_{\mu}\right)$ are non-zero and have opposite signs. Hence, if $p_{\mu}$ is $X$-hyperbolic (resp., $X$-elliptic), $p_{\mu}$ will be $Y$-elliptic (resp., $Y$-hyperbolic). This gives the remaining relations $e_{X}^{\mu}=h_{Y}^{\mu}, \quad h_{X}^{\mu}=e_{Y}^{\mu}$ in (7). 
As mentioned before, we still have to show that $\Sigma$ is finite. The fact that both second order derivatives $A\left(p_{\lambda}\right)$ and $A\left(p_{\mu}\right)$ are non-zero means that the $X$-trajectories that are tangent to the unit circle exhibit simple tangencies (as opposed to a contact of higher order). Using the compactness of $T$ one can then argue geometrically that the set of tangencies $(=\Sigma)$ must be discrete, hence finite. But instead of relying on geometric arguments, we give a short analytic proof that $\Sigma$ is finite.

Consider a point $p \in \Sigma$ and the map

$$
F(q)=(g(q),\langle X, \nabla g\rangle(q))
$$

defined for all $q$ sufficiently close to $p$. Observe that $F(q)=\left(\frac{1}{2}, 0\right)$ holds if and only if $q$ lies in $T$ and is a point where $X$ and $T$ are tangent. We want to show that, in a sufficiently small neighborhood of $p$ in $\mathbb{R}^{2}$, the only such point is $q=p$. Once this is done, compactness of $T$ implies that $\Sigma$ is finite. By the inverse function theorem, it suffices to show that $D F(p)$ is invertible. Writing $\left(a_{i j}\right)$ for the jacobian matrix of $F$ relative to the oriented basis $\left\{e_{1}=J X(p), e_{2}=X(p)\right\}$, we compute

$$
a_{11}=\langle J X(p), \nabla g(p)\rangle=-\left\langle H_{f} \nabla g, \nabla g\right\rangle,
$$

so that $a_{11}(p)$ has the value $-\lambda$ or $-\mu$, according to whether $p$ is a $\lambda$-point or a $\mu$-point. In particular, $a_{11}(p) \neq 0$. Also,

$$
a_{21}(p)=\langle X, \nabla g\rangle(p)=\left\langle J H_{f} \nabla g, \nabla g\right\rangle(p)=0,
$$

because $\nabla g(p)$ is an eigenvector of $H_{f}(p)$. Hence $\operatorname{det} D F(p)=a_{11} a_{22}$. It remains to check that $a_{22}(p) \neq 0$. But the quantity

$$
a_{22}(p)=\langle X, \nabla\langle X, \nabla g\rangle\rangle(p)
$$

was computed already. Indeed, by the remark following (8), $a_{22}(p)$ is simply $A(p)$ which, under the assumptions of the theorem, was found to be non-zero whether the point $p$ is a $\lambda$-point or a $\mu$-point. Hence $a_{22}(p) \neq 0$ and, as explained before, this is enough to show that $\Sigma$ is finite.

We can now finish the proof of Theorem 1. From (5),

$$
\operatorname{Ind}(Y, 0)=1+\frac{e_{Y}-h_{Y}}{2} .
$$

By (6) and (7),

$$
\begin{aligned}
& e_{Y}-h_{Y}=e_{Y}^{\lambda}+e_{Y}^{\mu}-h_{Y}^{\lambda}-h_{Y}^{\mu}= \\
& e_{X}^{\lambda}+h_{X}^{\mu}-h_{X}^{\lambda}-e_{X}^{\mu}= \\
& e_{X}^{\lambda}+h_{X}^{\mu}-h_{X}^{\lambda}-\left(h_{X}^{\lambda}+h_{X}^{\mu}-e_{X}^{\lambda}\right)= \\
& 2\left(e_{X}^{\lambda}-h_{X}^{\lambda}\right) .
\end{aligned}
$$


Similarly,

$$
\begin{aligned}
& e_{Y}-h_{Y}=e_{Y}^{\lambda}+e_{Y}^{\mu}-h_{Y}^{\lambda}-h_{Y}^{\mu}= \\
& e_{X}^{\lambda}+h_{X}^{\mu}-h_{X}^{\lambda}-e_{X}^{\mu}= \\
& \left(h_{X}^{\lambda}+h_{X}^{\mu}-e_{X}^{\mu}\right)+h_{X}^{\mu}-h_{X}^{\lambda}-e_{X}^{\mu}= \\
& 2\left(h_{X}^{\mu}-e_{X}^{\mu}\right) .
\end{aligned}
$$

From (2)-(4) and (19)-(21), we have

$$
\operatorname{Ind}\left(\frac{\partial^{2} f}{\partial \bar{z}^{2}}, 0\right)=2+e_{X}^{\lambda}-h_{X}^{\lambda}=2+h_{X}^{\mu}-e_{X}^{\mu} .
$$

In order to identify (22) with the index formulas given in the statement of the theorem, we appeal to (13) and (14). Indeed, one only needs to recall that $\lambda \geq \mu>0$, a $\lambda$-point $p_{\lambda}$ is $X$-elliptic (resp., $X$-hyperbolic) if and only if $A\left(p_{\lambda}\right)<0$ (resp., $A\left(p_{\lambda}\right)>0$ ), and a $\mu$-point $p_{\mu}$ is $X$-elliptic (resp., $X$-hyperbolic) if and only if $A\left(p_{\mu}\right)<0$ (resp., $A\left(p_{\mu}\right)>0$ ). This concludes the proof of Theorem 1.

\section{References}

[1] G. Bol, Über Nabelpunkte auf einer Eifläche, Math. Z. 49 (1944), 389-410.

[2] M. Dajczer, Submanifolds and Isometric Immersions, vol. 13, Mathematics Lecture Series, Publish or Perish, Inc. Houston, 1990.

[3] G. Garcia and C. Gutierrez, Ovaloids of $\mathbb{R}^{3}$ and their umbilics: A differential geometric approach, Jour. of Diff. Equations 168 (2000), 200-211.

[4] C. Gutierrez and J. Sotomayor, Lines of curvature, umbilic points and Carathéodory conjecture, Resenhas IME-USP 3 (1998), 291-322.

[5] H. L. Hamburger, Beweis einer Carathéodoryschen Vermütung. I, Ann. of Math. 41 (1940), 63-86.

[6] _ Beweis einer Carathéodoryschen Vermütung. II, III, Acta Math. 73, (1941), 174-332.

[7] P. Hartman, Ordinary Differential equations, John Wiley, New York, 1964.

[8] V. V. Ivanov, The analytic Carathéodory Conjecture, Siberian Math. J. 43 (2002), 251-322.

[9] T. Klotz, On G. Bol's proof of Carathéodory's conjecture, Comm. Pure Appl. Math. 12 (1959), 277-311.

[10] L. Lazarovici, Elliptic sectors in surface theory and the Carathéodory-Loewner conjectures, J. Diff. Geom. 55 (2000), 453-473.

[11] B. Smyth and F. Xavier, A sharp geometric estimate for the index of an umbilic on a smooth surface, Bull. London Math. Soc. 24 (1992), 176-180.

[12] B. Smyth and F. Xavier, Real solvability of the equation $\partial_{\bar{z}}^{2} f=\rho g$ and the topology of isolated umbilics, J. Geom. Anal. 8 (1998), 655-671.

[13] J. Sotomayor, Lines of curvature and an integral form of Mainardi-Codazzi equations, Acad. Brasil. de Ciências 68 (1996), 133-137.

[14] C. Titus, A proof of a conjecture of Loewner and of the conjecture of Carathéodory on umbilic points, Acta Mat. 131 (1973), 43-77.

Department of Mathematics University of Notre Dame, Notre Dame IN, 46635

E-mail address: xavier.1@nd.edu 